\title{
An Effective Noise Estimator for Use in Noise Reduction
}

\author{
Hag-Yong Han, Ho-Min Kwon, Sung-Mok Lee, Gi-Dong Lee and Bong-Soon Kang, Member, KIMICS
}

\begin{abstract}
Conventional noise reduction filtering schemes realize limited improvements of the peak signal-to-noise ratio (PSNR) in the low-level noisy images. The flatness degree and the edge information are effectively used to estimate the noise volume. We propose a noise estimator for reducing noise in the AWGN (additive white gaussian noise) corrupted images using three intermediate image maps (FGM(flatness gray map), FIM(flatness index map), NEM(noise estimate map)). The proposed noise estimator is fed into the conventional noise reduction filters as a pre-processor. The performance of noise reduction is tested in the various AWGN corrupted images.
\end{abstract}

Index Terms - noise reduction, noise estimation, lowlevel noise filtering

\section{INTRODUCTION}

Noise reduction in digital image applications has been a longstanding problem. Noise is introduced into digital imaging devices when the visual surrounding information is captured through image acquisition devices including optical lens, color filter arrays, photo sensors, etc. The primal cause is the lack of the dynamic range and sensitivity against the variant brightness. The problem affects the performance of image process, image storage, and image communication. Many researches have been reported to improve the image quality demanded from user expectations using diverse noise reduction schemes. AWGN corrupts digital video signals during their transmission through communication channels. For the noise reduction, several linear filtering approaches have been applied to the noise reduction [1].

Linear filters such as a mean filter and a Gaussian filter are amenable to analysis in the Fourier domain and require low complexity [2]. However, the blurring effect of the edge component occurs since the local brightness information is not accessible. In addition, such nonlinear filters including a median filter, a k-nearest neighbor (NN) algorithm, symmetric NN algorithms, and a sigma filter

Manuscript received December 14, 2010; revised January 10 2011; accepted January 17, 2011.

Hag-Yong Han, Sung-Mok Lee, Gi-Dong Lee, and Bong-Soon Kang are with the Dept. of Electronics Engineering, Dong-A University, Busan 604-714, South Korea (Tel: +82-51-200-7703, Fax: +82-51-200-7712, Email: bongsoon@dau.ac.kr)

Ho-Min Kwon is with the TruDef Research Inc., 9990 Mesa Rim Rd, Suite 160, San Diego, CA 92121, USA. have been reported to improve the blurring effect by means of adjusting individual pixels from the estimate of relations of pixels similarity and pixels adjacency. The design of nonlinear filters requires more sophisticated algorithms than that of linear filters. On the other hand, the nonlinear filters successfully reduce the noise while preserving the original image. In particular, Bilateral filter, SUSAN filter, and Non-Local (NL) means filter are reported useful to preserve the edge information [2]-[3]. Bilateral and SUSAN filters use the domain and range kernel for the spatial and intensity region respectively. NL-means filter pay the high computational cost for the pixel-by-pixel window matching. Other iterative adaptive smoothing and anisotropic diffusion noise reduction techniques have been applied into a "cartoon" [2].

These filtering approaches have shown the limited performance for practical applications because of the assumption that the Gaussian noise distribution or noise level is known. Given unknown noise characteristic, noise estimate has the challenges in terms of the accuracy and the computational complexity. In [4]-[5], the noise is estimated by computing the difference between the noisy image and the low-pass filtered image. The results show better performance when compared with each of the adaptive Gaussian filters and the estimate of the standard variance of the individual block. In [6], Shin et. al. proposed a blockbased noise estimate that makes use of the adaptive Gaussian filter combined with the blocks classification based on the standard deviation of their intensities. This method is reported the best performance among the Lee and Hoppel[7], Olsen[8], Rank et al.[9] methods. However, the performance of the noise estimate can be varied according to the block size. Given the noisy image containing much more information of the edge component, the noise volume can be incorrectly increased. For example of a noise reduction, we used seven popular linear and nonlinear filters of an average filter, a k-NN algorithm, a symmetric NN algorithm, a global sigma filter, a local sigma filter, a Gaussian filter, and a median filter. We added the various AWGNs to a "Lenna" image. Seven different conventional filters were employed to compare the PSNRs between the original image and the corrupted input images, as shown in Figure 1. The linear filters and the nonlinear filters showed the higher PSNRs than that of the input image around at the 6 standard deviation above. However, around at the 6 standard deviation below, the 
PSNRs of the filtered images are lower than that of the input image. This implies that the AWGN is globally embedded in the image. If the local noise characteristic can be estimated in the image, more accurate noise estimate can be used to improve the performance of the above seven filters.

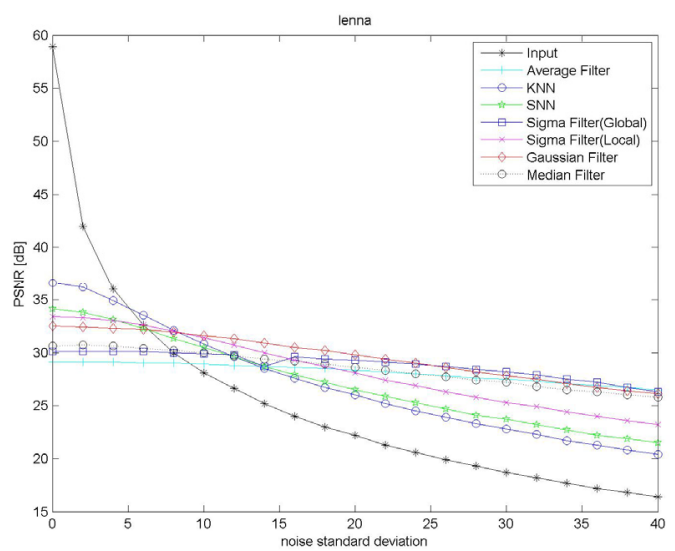

Fig. 1 Performance comparisons of conventional noisereduction techniques for the low-level noise image in the "Lenna" image.

In order to increase the low-level noise reduction performance of the conventional filters, we propose a noise estimate which makes use of the local noise characteristic and use three intermediate image maps (FGM, FIM, NEM). The flatness degree and the edge information are efficiently used to estimate the low-level AWGN volume. Combining with the conventional noise reduction filters, the proposed noise estimator benefits from the low-level noise reduction as a preprocessor.

\section{PROPOSED NOISE ESTIMATOR}

The proposed noise estimator consists of three stages. In the first stage, we prepare the flatness gray map (FGM) image. Given the noisy image $x(m, n)$, the FGM image is calculated by

$$
\begin{gathered}
F G M_{i}=\frac{1}{L-1} \sum_{m, n}\left|x^{i}(m, n)-x_{c}^{i}(m, n)\right| \\
m \in[1, M], n \in[1, N]
\end{gathered}
$$

where $\mathrm{L}$ is the mask size, $\mathrm{M}$ is the width, $\mathrm{N}$ is the height, $x_{c}^{i}(m, n)$ is the central pixel, $x^{i}(m, n)$ is the neighboring pixel in the ith mask window. The FGM image provides the flatness degree and the edge information from the underlying image.
In the second stage, the histogram of the pixel bright values ranging from 0 to 255 is calculated to obtain its cumulative distribution function (CDF) $f\left(F G M_{i}\right)$. To deal with the dynamic variance of the AWGN volume and image quality, we empirically chose the $80 \%$ threshold as optimum. Figure 3 shows the results of the validating threshold selection ranging from 0 to $100 \%$ with 4 different images. Although the number of the testing images is limited, For about 10 standard deviation of AWGN noise estimate, the $80 \%$ threshold resulted in the best. The $80 \%$ is the threshold to separate the image pixels into the flat region and the non-flat region as shown in Eq. (2).

$$
\delta^{i}(m, n)=\left\{\begin{array}{l}
1, \text { if } f\left(F G M_{i}\right) \geq 0.8 \\
0, \text { otherwise }
\end{array}\right.
$$

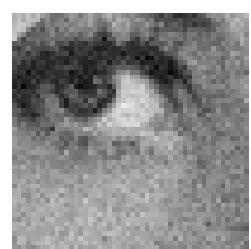

(a)

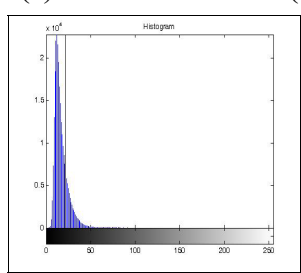

(d)

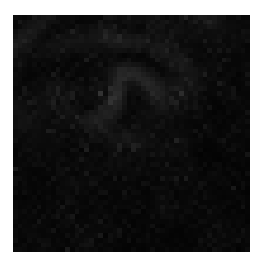

(b)

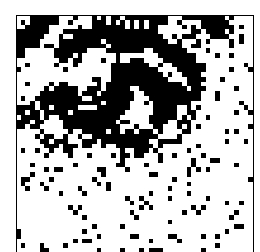

(c)

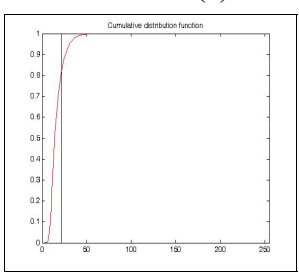

(e)

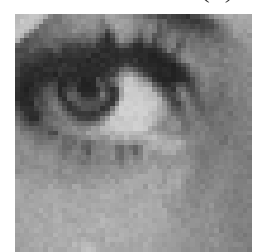

(f)

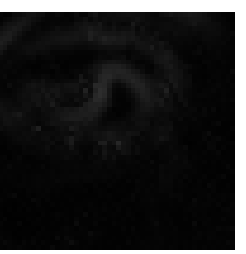

(g)

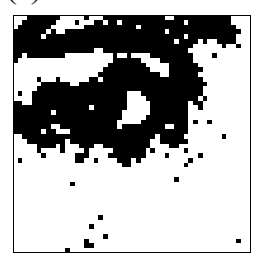

(h)

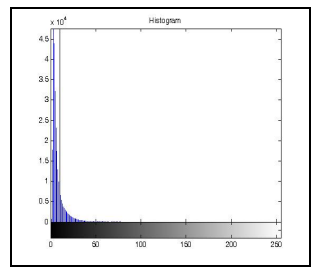

(i)

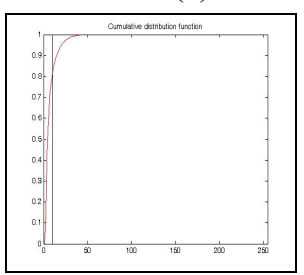

(j)
Fig. 2 Upper images with PSNR=26dB: (a) input noisy image, (b) the FGM image, (c) the FIM image, (d) the histogram of the FGM, and (e) the CDF of the FGM. Lower images with $P S N R=38 d B$ : (f) input noisy image, (g) the FGM image, (h) the FIM image, (i) the histogram of the FGM, and (j) the CDF of the FGM. 
The flat region includes the edge information as well as the noise information. We denote the $\delta^{i}(m, n)$ image by the flatness index map (FIM). Figure 2(a) and (f) show left eyes of the "Lenna" image corrupted by the different volume with PSNRs (26dB and $38 \mathrm{~dB}$, respectively), Figure 2(b) and (g) show the FGMs, and Figure 2 (c) and (h) show the FIMs. Figures 2(d) and (i) show the histogram of the FGMs, and Figure 2(e) and (j) show the cumulative distribution functions of the FGMs. The vertical line indicates the $80 \%$ threshold.

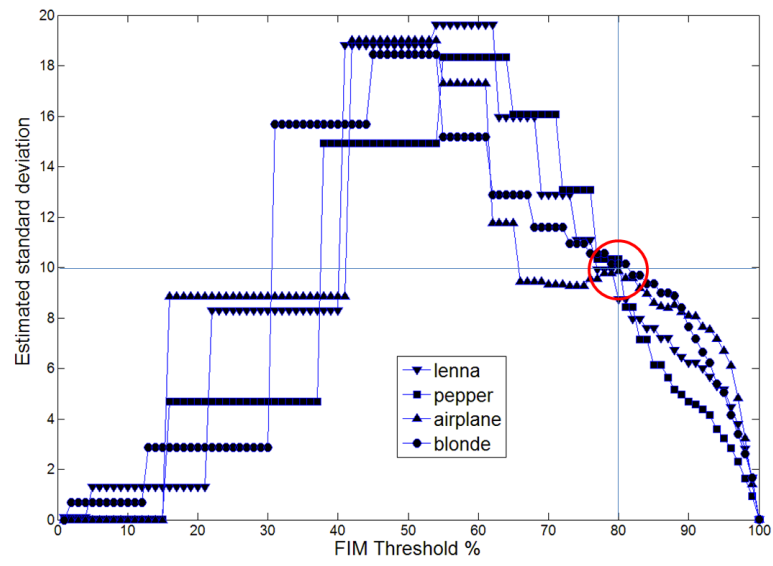

Fig. 3 Performance comparison according to the FIM threshold \%

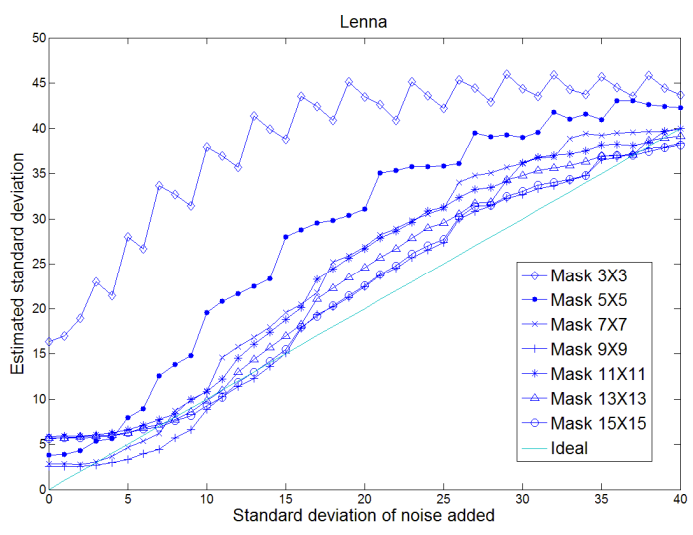

Fig. 4 Performance comparison according to the various mask size.

All images size used in this letter are $512 \times 512$ size. Every mask based image processing methods take effect by the mask size in the processing. In this letter, we can apply with the various mask size only in the FGM image generation processing step. Figure 4 shows the estimation performance according to the various mask size. Because of the central pixel represents the flatness degree in that area. To large the mask size, to better performance up to 9x9 size, But too large mask is rather harmful for the performance. So it is required that the processing with the optimal mask size and normalizing the input image size to the $512 \times 512$ size. The $9 \times 9$ mask size performed the best in the "Lenna" image.

In the third stage, we estimate the noise volume threshold $\delta^{i}(m, n)$ in the FIM. If the number of $\delta^{i}(m, n)$ is less than 3 and $x^{i}(m, n)=0, \delta^{i}(m, n)$ is assumed as the noise pixel. The noise estimate map (NEM) is calculated by Eq.(3) and is for removing the edge components in the FIM image and generating the NEM image which only contain the noise component. Noise pixel composed of the small pixels and pixel threshold factor (pixel_th) is the empirical value. The reason using the static $3 \times 3$ mask size in Eq.(3) is for counting the small pixel and $3 \times 3$ mask size is the minimum size including the central pixel. Figure 5 shows the optimal estimation results in the 3 pixel for the noise standard deviation 10 gaussian added noise. So we select the pixel threshold with 3 .
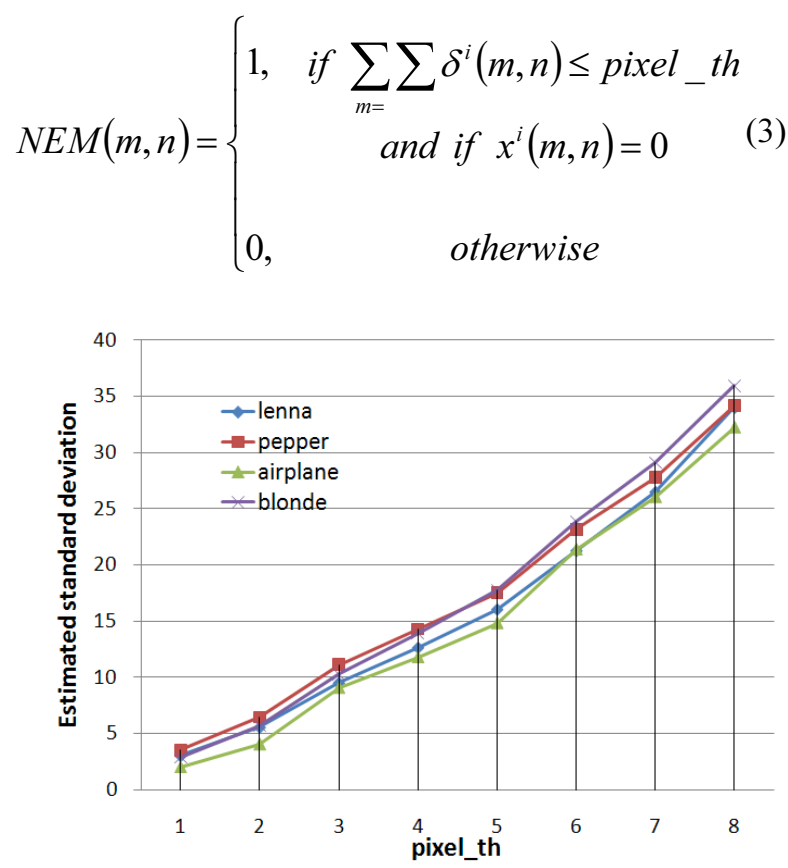

Fig. 5 Performance comparison of the noise estimator for the different mask sizes and noise volume.

$$
N V=\alpha \times \frac{\sum_{m, n} N E M(m, n)}{M \times N} \times 100
$$

where $\alpha$ is the conversion factor of standard deviation for the noise volume. We empirically choose $\alpha$ as 2.47 as optimum. 


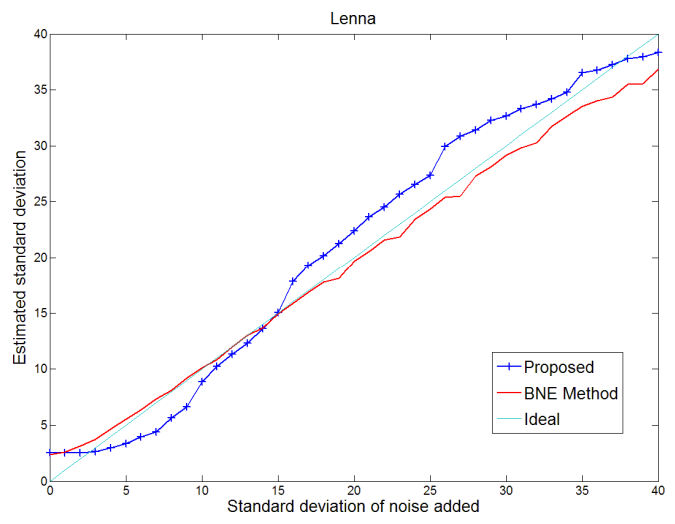

Fig. 6 Performance comparison with the BNE and proposed method.

As typical noise estimator, there is the standardized median of absolute deviation from the median (MAD [10]). But MAD approach is base on the wavelet and assumes that the coefficients of the finest decomposition level are associated only to the noise and uses the median of absolute value of these coefficients for variance estimation [11]. Instead of comparison with MAD, we compare with the block-based noise estimation method proposed by Shin et al. which is very similar approach to this method. Figure 6 shows the performance comparison with the BNE.

Figure 7 depicts simulation results in the standard deviations 10 and 30 of additive noise volume respectively for the four test images. Figure $7(a)(e)$ is input noisy images. Figures $7(\mathrm{~b})(\mathrm{f})$ and $(\mathrm{c})(\mathrm{g})$ are the corresponding the FGMs and the FIMs, respectively. Figure $7(d)(h)$ is the resulting NEMs. From the figures, we can see that the flatness degree decreases in the FGMs and FIMs as the standard deviation increases. The edge information is removed by using Eq. (3) and the estimated noise volume remains increasingly in the NEMs.

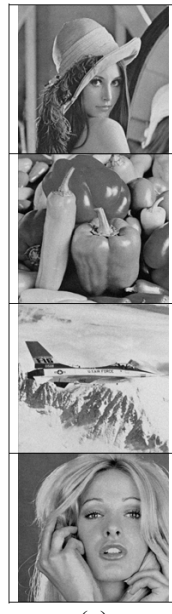

(a)

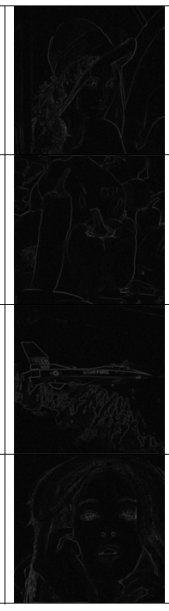

(b)

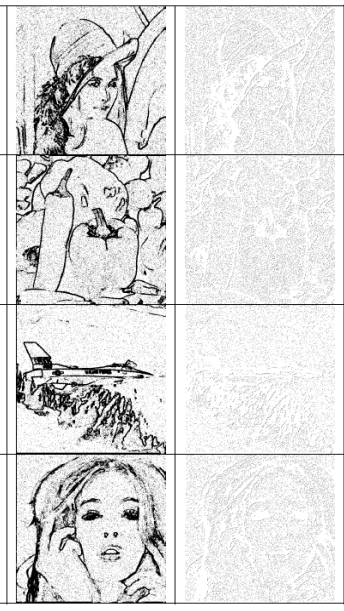

(c) (d)

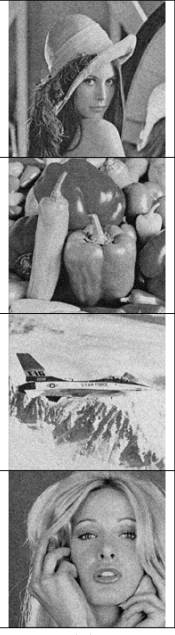

(e)

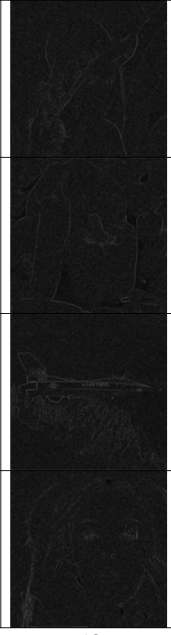

(f)

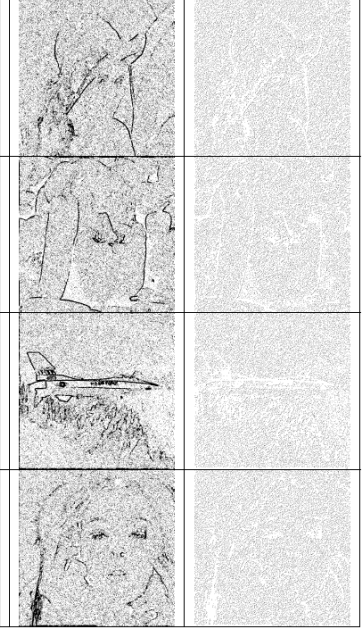

(g) (h)
Fig. 7 Simulation results in the standard deviations 10 and 30 of the additive noise volume: (a)(e) input noisy image, (b)(f) the FGMs, (c)(g) the FIMs, and (d)(h) the NEMs.

\section{RESULTS AND DISCUSSION}

The noise reduction filtering was simulated using the proposed noise estimator in the "Lenna" image. We made use of the seven conventional filters as shown in Figure 1. The standard deviations range from 0 to 40 . Figure 8 shows the improved performance of the noise reduction. The proposed noise estimator fed into the conventional filters significantly improved the PSNRs around at the 8 standard deviation or smaller. Around at the 8 standard deviation or higher, the performance of the noise reduction filtering is identical with that as shown in Figure 1. The proposed noise estimator measures effectively the additive noise volume and, thereby, reduces the low-level noise.

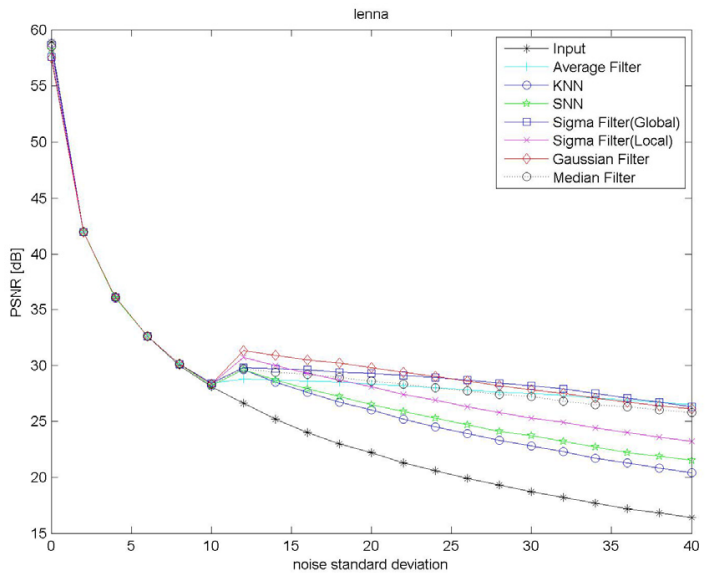

Fig. 8 Improved performance of conventional noisereduction techniques for the low-level noise image with the proposed method. 


\section{CONCLUSIONS}

In the paper, we proposed the noise estimator for filtering based noise reduction. The proposed flatness information and its consecutive binary mapping utilization were capable of removing the edge information and estimating the noise volume in the various noisy images. The 9x9 mask size performed the best to estimate the additive noise volume. The simulation results show that when the conventional noise reduction filtering techniques combined with the proposed noise estimator significantly improved the PSNRs in the low-level noisy images as well as in the high-level noisy images.

\section{ACKNOWLEDGMENT}

This work was supported by the research funds from Dong-A University.

\section{REFERENCES}

[1] R. C. Gonzalez, R. E. Woods and S. L. Eddins, "Digital Image Processing using MATLAB," Pearson Prentice Hall, pp. 89-107, 2004."

[2] A. Buades, B. Coll, and J.M. Morel, "A non local algorithm for image denoising," IEEE Computer Vision and Pattern Recognition 2005, Vol. 2, pp. 60-65, 2005

[3] S.M. Smith and J.M. Brady, "SUSAN-A New Approach to Low Level Image Processing," International Journal of Computer Vision, Vol. 23, No. 1, pp. 45-78, May 1997.

[4] R.C. Bilcu and M. Vehvilainen, "A New Method for Noise Estimation in Images," Proc. IEEE EURASIP International Workshop on Nonlinear Signal and Image Processing, Sapporo, Japan, May 2005.

[5] S.C. Tai and S.M. Yang, "A Fast Method for Image Noise Estimation Using Laplacian Operator and Adaptive Edge Detection," ISCCSP 2008, Malta, March 2008.

[6] D.-H. Shin, R.-H Park, S. Yang, and J.-H. Jung, "Block-Based Noise Estimation Using Adaptive Gaussian Filtering," IEEE Trans. on Consumer Electronics, Vol. 51, No. 1, pp. 218-226, 2005.

[7] J.S. Lee and K. Hoppel, "Noise modeling and estimation of remotely-sensed image," in Proc. Int. Geoscience and Remote Sensing, Vancouver, Canada,, Vol. 2, pp. 1005-1008, June 1989.

[8] S. I. Olsen, "Estimation of noise in images: An evaluation," Graphical Models and Image Process., Vol. 55, pp. 319-329, July 1993.

[9] K. Rank, M. Lendl, and R. Unbehauen, "Estimation of image noise variance," IEE Proc. Vis. Image Signal Processing, Vol. 146, pp. 80-84, Apr. 1999.

[10] D.L. Donoho, "De-Noising by Soft-Thresholding," IEEE Transactions on Information Theory, Vol. 41, No. 3, May 1995.

[11] M. Hashemi, S. Beheshti, "Adaptive Noise Variance Estimation in BayesShrink," IEEE Signal Processing Letters, Vol. 17, No.1, January 2010 .

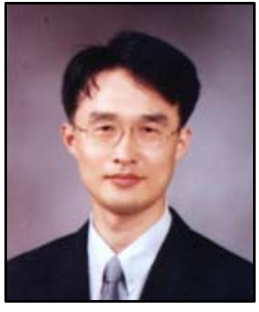

Hag-Yong Han received the B.S., M.S. and Ph.D. degrees in electronics engineering from Dong-A University, Busan, Korea, in 1994, 1998 and 2004, respectively. He had worked as Chief of R\&D at the Easy Harmony Co. Ltd. Busan, Korea, in 2001, and a post doctoral researcher at the Pusan National University, Busan Korea, in 2006-2007, respectively. He now is the research professor of the Multimedia Research Center of the Dong-A University. His research interests include pattern recognition, audio / image / video processing, DSP application

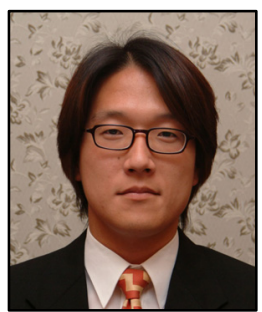

Ho-Min Kwon received the B.S. and M.S degrees in electronics engineering from DongA University, Busan, Korea in 2002 and 2004 , respectively, and the Ph.D. degree from Arizona State University in 2009. While at ASU, he worked and published in J-DSP, acoustic sensing in wireless sensor networks and voice scene characterization. He had held summer internship appointments at DynaSig Co. in 2006-2007. He is a senior research engineer at TruDef Research, Inc. where his work focuses on the region of interest based video quality improvements and image enhancement algorithms.

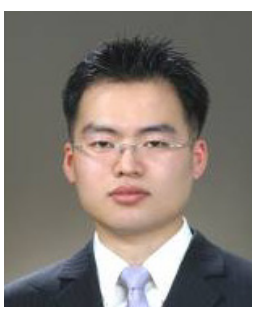

Sung-Mok Lee received the B.S and M.S degrees in electronic engineering from Dong-A University, Busan, Korea, in 2005 and 2007, respectively. He is currently working toward his $\mathrm{Ph} . \mathrm{D}$. degree at the university. His research interests include digital camera processing systems, VLSI architecture design and image processing

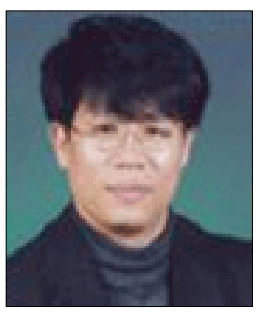

Gi-Dong Lee received the B.S., M.S. and Ph.D. degrees in electronics engineering from Pusan National University, Busan, Korea, in 1989, 1991 and 2000, respectively. He had worked at LCD R\&D center in Samsung SDI, Korea, in 1991-1997, and a Research Fellow at Liquid Crystal Institute, Kent State University, USA, in 2001-2003, respectively. Since 2004, he has been with the department of electronics engineering, Dong-A University, Busan, Korea. His research interests include display devices.

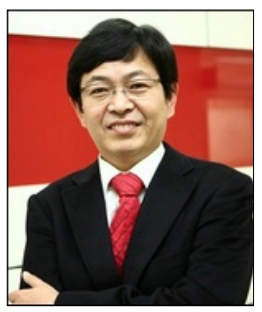

Bong-Soon Kang received the B.S. degree in electronics engineering from Yonsei University, Seoul, Korea, in 1985, and the M.S. degree in electrical engineering. from University of Pennsylvania, Pennsylvania, USA, in 1987, and the Ph.D. degree in electrical and computer engineering from Drexel University, Philadelphia, USA, in 1990. From Dec. 1989 to Feb. 1999, he had worked as a senior staff researcher at Samsung Electronics Co. Ltd., Kihung, Korea. Since March 1999, he has been with the department of electronics engineering, Dong-A University, Busan, Korea. He is the director of the Multimedia Research Center of the university. His research interests include image processing, hardware architecture designs, and wireless communications. He was honored as a 2007 winner of the Chester Sall Award for the 1st place best paper in the IEEE Transactions on Consumer Electronics on Jan. 2009. 